\title{
On Approximately Inner Automorphisms of Certain Crossed Product C \# - Algebras
}

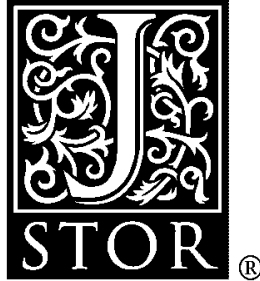

\author{
Marius D\#d\#rlat; Cornel Pasnicu \\ Proceedings of the American Mathematical Society, Vol. 110, No. 2. (Oct., 1990), pp. 383-385.
}

Stable URL:

http://links.jstor.org/sici?sici=0002-9939\%28199010\%29110\%3A2\%3C383\%3AOAIAOC\%3E2.0.CO\%3B2-J

Proceedings of the American Mathematical Society is currently published by American Mathematical Society.

Your use of the JSTOR archive indicates your acceptance of JSTOR's Terms and Conditions of Use, available at http://www.jstor.org/about/terms.html. JSTOR's Terms and Conditions of Use provides, in part, that unless you have obtained prior permission, you may not download an entire issue of a journal or multiple copies of articles, and you may use content in the JSTOR archive only for your personal, non-commercial use.

Please contact the publisher regarding any further use of this work. Publisher contact information may be obtained at http://www.jstor.org/journals/ams.html.

Each copy of any part of a JSTOR transmission must contain the same copyright notice that appears on the screen or printed page of such transmission.

The JSTOR Archive is a trusted digital repository providing for long-term preservation and access to leading academic journals and scholarly literature from around the world. The Archive is supported by libraries, scholarly societies, publishers, and foundations. It is an initiative of JSTOR, a not-for-profit organization with a mission to help the scholarly community take advantage of advances in technology. For more information regarding JSTOR, please contact support@jstor.org. 


\title{
ON APPROXIMATELY INNER AUTOMORPHISMS OF CERTAIN CROSSED PRODUCT $C^{*}$-ALGEBRAS
}

\author{
MARIUS DĂDĂRLAT AND CORNEL PASNICU
}

(Communicated by Paul S. Muhly)

\begin{abstract}
Let $G$ be a compact connected topological group having a dense subgroup isomorphic to $\mathbf{Z}$. Let $C(G) \underset{\propto}{\rtimes \mathbf{Z}}$ be the crossed product $C^{*}$-algebra of $C(G)$ with $\mathbf{Z}$, where $\mathbf{Z}$ acts on $G$ by rotations. Automorphisms of $C(G) \underset{\propto}{\underset{Z}{Z}}$ leaving invariant the canonical copy of $C(G)$ are shown to be approximately inner iff they act trivially on $K_{1}(C(G) \underset{\propto}{\rtimes} \mathbf{Z})$.
\end{abstract}

Let $G$ be a compact abelian topological group. An element $s \in G$ is called a generator if the group algebraically generated by $s$ is dense in $G$. $G$ is called monothetic if it has at least one generator. If in addition $G$ is connected, this is equivalent to saying that the topology of $G$ has a base of cardinality $\leq c$. Moreover if $G$ is second countable then the set of generators is measurable and its Haar measure equals 1. (See [4], Theorems 24.15, 24.27.)

From now on, $G$ is a monothetic compact connected infinite topological group and $s \in G$ is a fixed generator. Let $A=C(G)$ be the $C^{*}$-algebra of all complex-valued continuous functions on $G$. We consider the action $\propto: \mathbf{Z} \rightarrow \operatorname{Aut}(A)$ given by

$$
\left(\propto_{k}(a)\right)(x)=a\left(s^{-k} x\right), \quad \text { for } a \in A, \quad x \in G
$$

and the corresponding crossed product $C^{*}$-algebra $A \rtimes \mathbf{Z}$ (see [5, 8]). Denote by $\operatorname{Aut}_{A}(A \underset{\propto}{\rtimes \mathbf{Z})}$ the closed subgroup

$$
\{\beta \in \operatorname{Aut}(A \underset{\propto}{\rtimes} \mathbf{Z}): \beta(A)=A\}
$$

where $\operatorname{Aut}(A \rtimes \mathbf{Z})$ has the topology of pointwise norm convergence. Note that $\operatorname{Aut}_{A}(A \underset{\propto}{\rtimes} \mathbf{Z}) \stackrel{\propto}{=}\{\beta \in \operatorname{Aut}(A \underset{\propto}{\rtimes} \mathbf{Z}): \beta(A) \subset A\}$, since $A$ is a maximal abelian self-adjoint subalgebra in $A \underset{\propto}{\propto} \mathrm{Z}$ (see [8], Proposition 4.14). We prove the following.

Received by the editors May 4, 1989; presented at the OATE 2 Conference, Craiova, Romania, August 28-September 8, 1989, organized by INCREST.

1980 Mathematics Subject Classification (1985 Revision). Primary 46L40; Secondary 46L80. 
1. Theorem. An automorphism $\beta \in \operatorname{Aut}_{A}(A \underset{\propto}{\rtimes} \mathbf{Z})$ is approximately inner iff $\beta$ induces the identity automorphism of $K_{1}(A \underset{\propto}{\rtimes} \mathbf{\complement})$.

For $G$ isomorphic to the one-dimensional torus $\mathbf{T}$, the corresponding result is due to Brenken [2].

The proof uses the description of $\operatorname{Aut}_{A}(\underset{\propto}{\rtimes} \mathbf{Z})$ which follows from more general results [3, Theorem 2.8].

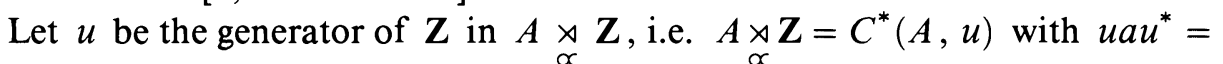
$\propto_{1}(a)$ for $a \in A$. Then each $\beta \in \operatorname{Aut}_{A}(A \underset{\propto}{\rtimes} \mathbf{Z})^{\propto}$ is given by a unique triplet $(b, x, q) \in U(A) \times G \times\{-1,1\}$ such that $\beta(u)=b u^{q}$ and $\beta(a)(y)=a\left(x y^{q}\right)$ for $a \in A, y \in G$. Here $U(A)$ denotes the unitary group of $A$ (with the norm topology) and the correspondence $\beta \leftrightarrow(b, x, q)$ is a homeomorphism. It follows by ([3], Lemma 2.4) that such an automorphism is inner iff $q=1$, $x=s^{k}$ for some $k \in \mathbf{Z}$ and $b$ has the form $w(\cdot) w^{*}\left(s^{-1} \cdot\right)$ for some $w \in U(A)$. In this case $\beta(t)=w u^{-k} t u^{k} w^{*}, t \in A \underset{\propto}{\rtimes} \mathbf{Z}$. Therefore if $\beta \in \operatorname{Aut}_{A}(A \underset{\propto}{\rtimes} \mathbf{Z})$ is given by $(b, x, q)$ then $\beta$ is approximately inner provided that $q=1$ and that $b$ is in the closure of the set

$$
\left\{w(\cdot) w^{*}\left(s^{-1} \cdot\right): w \in U(A)\right\}
$$

Indeed, if $w_{n}(\cdot) w_{n}^{*}\left(s^{-1} \cdot\right)$ converges to $b$ in $U(A)$ and $s^{k_{n}}$ converges to $x$ in $G$ then, $\operatorname{ad}\left(w_{n} u^{-k_{n}}\right)$ converges to $\beta$ in $\operatorname{Aut}_{A}(A \underset{\propto}{\rtimes} \mathbf{Z})$.

2. Lemma. Let $\beta \in \operatorname{Aut}_{A}(A \underset{\propto}{\rtimes} \mathbf{Z})$ be given by $(b, x, q)$. If $\beta$ induces the identity automorphism of $K_{1}(A \underset{\propto}{\propto} \mathbf{Z})$ ) then $q=1$ and $b \in U_{0}(A)$ (the connected component of the identity in $U(A))$.

Proof. Since $G$ is connected it follows that $\propto_{1}$ induces the identity automorphism of $K_{1}(A)$. Using the Pimsner-Voiculescu exact sequence [6] one sees that the canonical map $K_{1}(A) \rightarrow K_{1}(A \underset{\propto}{\rtimes} \mathbf{Z})$ is injective. The obvious map $\pi^{1}(G):=[G, \mathbf{T}] \rightarrow K_{1}(A)$ is also injective (use for instance the determinant map). Consequently, if $a \in U(A)$ then $a \in U_{0}(A)$ iff $[a]=0$ in $K_{1}(A \underset{\propto}{\rtimes} \mathbf{Z})$.

For $\gamma \in \widehat{G}$ (the Pontrjagin dual of $G$ ) we have $\beta(\gamma)=\gamma(x) \gamma^{q}$. Therefore $[\gamma]=\left[\gamma^{q}\right]$ in $K_{1}(A \underset{\propto}{\rtimes} \mathbf{Z})$ and by the above remarks $\gamma$ is homotopic to $\gamma^{q}$ as maps $G \rightarrow \mathbf{T}$. By a result of Scheffer [7] this is possible only if $q=1$. The equation $\beta(u)=b u$ implies that $[\beta(u)]=[b]+[u]$ in $K_{1}(A \underset{\propto}{\rtimes} \mathbf{Z})$ hence using the hypothesis on $\beta$ and the above remarks we find that $b \in U_{0}(A)$.

3. Lemma. The map $w \rightarrow w(\cdot) w^{*}\left(s^{-1} \cdot\right)$ from $U(A)$ to $U_{0}(A)$ has dense range (compare with Theorem 4 in [2]).

Proof. Let $A_{s}=\left\{a(\cdot)-a\left(s^{-1} \cdot\right), a \in A\right\}$. Our first aim is to prove that $A_{s}+\mathbf{C} .1$ is a dense (linear, self-adjoint) subspace of $A$. This is accomplished by showing 
that it contains the *-subalgebra of $C(G)$ generated by the characters of $G$ (which is dense in $C(G)$ by the Stone-Weierstrass Theorem). We use the fact that

$$
S=\{\chi(s), \chi \in \widehat{G} \backslash\{1\}\}
$$

is a dense subset of $\mathbf{T}$ and $1 \notin S$ (see [4], Theorem 25.11). Thus if $\gamma \in \widehat{G} \backslash\{1\}$ then $a=\left(1-\gamma\left(s^{-1}\right)\right)^{-1} \gamma$ is such that $\gamma=a(\cdot)-a\left(s^{-1} \cdot\right) \in A_{s}$.

Any $v \in U_{0}(A)$ has the form $v=\exp (i h)$ for some $h \in C(G, \mathbf{R})$. By the above discussion we can find $a \in C(G, \mathbf{R})$ and $\lambda \in \mathbf{R}$ such that $a(\cdot)-a\left(s^{-1} \cdot\right)+\lambda$ is arbitrarily close to $h$ in norm. Also there is $\gamma \in \widehat{G} \backslash\{1\}$ such that $\left|e^{i \lambda}-\gamma(s)\right|$ is arbitrarily small. Then for $w=\gamma \exp (i a)$,

$$
w(\cdot) w^{*}\left(s^{-1} \cdot\right)=\gamma(s) \cdot \exp i\left(a(\cdot)-a\left(s^{-1} \cdot\right)\right)
$$

will approximate $v$ as well as we want.

Proof of the theorem. If $\beta \in \operatorname{Aut}_{A}(A \underset{\propto}{\rtimes} \mathbf{Z})$ given by $(b, x, q)$ induces the identity automorphism of $K_{1}(A \underset{\propto}{\rtimes} \mathbf{Z})$ then by Lemma $2, b \in U_{0}(A)$ and $q=1$. Using Lemma 3 we can find a sequence $w_{n} \in U(A)$ such that $w_{n}(\cdot) w_{n}^{*}\left(s^{-1} \cdot\right)$ converges to $b$ in $U_{0}(A)$. The discussion before Lemma 2 shows that $\beta$ is approximately inner. The reverse implication is a general fact.

\section{REFERENCES}

1. B. Blackadar, $K$-theory for operator algebras, MSRI Publications, Series 5, Springer-Verlag, New York, 1986.

2. B. Brenken, Approximately inner automorphisms of the irrational rotation algebra, C. R. Math. Rep. Acad. Sci. Canada VII (1985).

3. M. Dădărlat and C. Pasnicu, On certain automorphisms of reduced crossed products with discrete groups, J. Operator Theory 23 (1990), 179-193.

4. E. Hewit and K. A. Ross, Abstract harmonic analysis, Springer-Verlag, Berlin, 1983.

5. G. K. Pedersen, $C^{*}$-algebras and their automorphism groups, Academic Press, New York, 1979.

6. M. V. Pimsner and D. Voiculescu, Exact sequences for $K$-groups and Ext-groups of certain crossed product $C^{*}$-algebras, J. Operator Theory 4 (1980), 93-118.

7. W. Scheffer, Maps between topological groups that are homotopic to homomorphisms, Proc. Amer. Math. Soc. 33 (1972), 562-567.

8. G. Zeller-Meyer, Produits croisés d'une $C^{*}$-algèbre par un grôupe d'automorphismes, J. Math. Pures Appl. (9) 47 (1968), 101-239.

INCREST, Department of Mathematics, Bd. Pácil 220, 79622 Bucharest, Romania 
http://www.jstor.org

\section{LINKED CITATIONS \\ - Page 1 of 1 -}

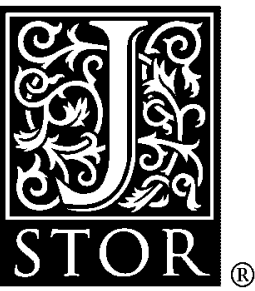

You have printed the following article:

On Approximately Inner Automorphisms of Certain Crossed Product C \# - Algebras

Marius D\#d\#rlat; Cornel Pasnicu

Proceedings of the American Mathematical Society, Vol. 110, No. 2. (Oct., 1990), pp. 383-385.

Stable URL:

http://links.jstor.org/sici?sici=0002-9939\%28199010\%29110\%3A2\%3C383\%3AOAIAOC\%3E2.0.CO\%3B2-J

This article references the following linked citations. If you are trying to access articles from an off-campus location, you may be required to first logon via your library web site to access JSTOR. Please visit your library's website or contact a librarian to learn about options for remote access to JSTOR.

\section{References}

\footnotetext{
${ }^{7}$ Maps Between Topological Groups That are Homotopic to Homomorphisms

Wladimiro Scheffer

Proceedings of the American Mathematical Society, Vol. 33, No. 2. (Jun., 1972), pp. 562-567.

Stable URL:

http://links.jstor.org/sici?sici=0002-9939\%28197206\%2933\%3A2\%3C562\%3AMBTGTA\%3E2.0.CO\%3B2-D
}

NOTE: The reference numbering from the original has been maintained in this citation list. 